\title{
Monogenoids from Freshwater Fish in Italy, with Comments on Alien Species
}

\author{
Paolo Galli, ${ }^{1,3}$ Giovanni Strona,,${ }^{1}$ Francesca Benzoni,,${ }^{1}$ Giuseppe Crosa, ${ }^{2}$ and \\ FABRIZIO STEFANI ${ }^{1}$ \\ ${ }^{1}$ Department of Biotechnology and Biosciences, University of Milano-Bicocca, Piazza della Scienza 2, \\ 20126 Milano, Italy (e-mail: paolo.galli@unimib.it), and \\ ${ }^{2}$ Department of Biotechnology and Molecular Sciences, University of Insubria, via Dunant 3, 21100 Varese, Italy
}

?1 ABSTRACT: Sixteen species of both native and alien fish were collected and examined for monogenoids from September 2001 to March 2004 in bodies of water located within fishing areas of the Po River (northern Italy). A checklist of monogenoids was compiled and integrated with literature reports of Italian monogenoids, updating the number of known Italian freshwater monogenoids to 35, with 15 new host or locality records (or both). Parasitological data of monogenoids on native and alien demonstrate the ability of alien monogenoids to colonize native hosts. The process of invasion of nonnative monogenoids in Italy started about $30 \mathrm{yr}$ ago and is still ongoing: 17 of the 35 monogenoids now present in Italian freshwaters are considered alien ( 5 species of monogenoids arrived in Italy from America, 11 from Central Europe, and 1 from Eurasia), 2 are considered native, and the remaining 16 are considered of unknown origin because of our limited knowledge concerning the original distribution of monogenoids.

KEY WORDS: alien parasites, Italy, Monogenoidea, introduced species.

In Italy there are roughly 600 taxa of fish classified as species or subspecies (Amori et al., 1993). Of these, 82 are freshwater taxa, in that their biological and ecological characteristics allow them to be defined as stenohaline freshwater, obligatory migratory euryhaline species, or nonobligatory migratory euryhaline species (Zerunian and De Ruosi, 2002). Italy, like other Mediterranean countries, has a rich endemic freshwater fish fauna, which is mainly the result of paleogeographic events in the Mediterranean area during the Late Miocene and Pleistocene ages. The present distribution pattern is the result of Pleistocene events, during the last Wurm glaciation (Bianco, 1998). Nevertheless, transfers have also altered the distribution pattern; among 82 species of freshwater fish, 34 are considered aliens (Bianco and Ketmaier, 2001). Despite high fish biodiversity in Italy, few studies have investigated the presence of monogenoids, the largest group of fish parasites. Before 2002, only 8 monogenoids from freshwater fish in Italy had been reported (Molnar and Ghittino, 1977; Gattaponi and Corallini Sorcetti, 1982; Bona et al., 1995). Sustained efforts to study these organisms date from the work of Galli et al. (2001, 2002, 2003, 2005). The importance of studying parasites is due to a rapid evolution of freshwater fish communities. In the past $30 \mathrm{yr}$ there have been processes of "padanization," with transplantation of native species from northern to central Italy, followed by "danubization," with introduction of Danubian

\footnotetext{
${ }^{3}$ Corresponding author.
}

species throughout Italy and, more recently, establishment of Iberian, Albanian, Eastern Asian, North American, and African elements (Bianco and Ketmaier, 2001). When fish are introduced into a new territory their parasites may also follow, forming a sort of biotic unit called "symbiota" (Galli et al., 2005). The success of establishment of parasites (both in new geographical areas and new hosts) has been described in detail by different authors (Dogiel, 1964; Bauer, 1991; Esch and Fernández, 1993; Vitousek et al., 1997; Torchin et al., 2001, 2002, 2003; Arndt et al., 2002; Bauer et al., 2002; Galli et al., 2003), and may depend on: (1) the complexity of the parasite life cycle: heteroxenous parasites with a life cycle that requires more than one host have a lower probability of introduction and establishment (Bauer, 1991); (2) the age of the host: according to Bauer (1991), younger fish, fry or fingerlings, are less infected than adults; (3) how often the host population has been introduced in the new area; (4) global climate change: according to Eaton and Scheller (1996) climate change might result in the range expansion of warm-water fish.

This paper provides a historical review of the study of the monogenoids from freshwater fish in Italy, presents an updated parasite-host checklist, and includes some comments regarding presence of alien parasites.

\section{MATERIALS AND METHODS}

A checklist of monogenoids parasitizing Italian freshwater fish was compiled using both past literature and original data. For this study, fish were collected during 
Table 1. Checklist of Italian monogenoids with their hosts and locations in Italy.

\begin{tabular}{|c|c|c|c|}
\hline Parasite & Host & Location & Previous locality records \\
\hline \multicolumn{4}{|l|}{ Dactylogyridae } \\
\hline Dactylogyrus alatus Linstow, 1878 f. typica & Rutilus aula & Como Lake & None \\
\hline Dactylogyrus anchoratus (Dujardin, 1845) & Carassius carassius & Varese Lake & Galli et al., 2002 \\
\hline \multirow[t]{2}{*}{ Dactylogyrus caballeroi Prost, 1960} & Rutilus aula & Como Lake & None \\
\hline & Rutilus aula & Ticino River & None \\
\hline Dactylogyrus crucifer Wagener, 1857 & Rutilus pigus & Como Lake & None \\
\hline Dactylogyrus difformis Wagener, 1857 & Scardinius erythrophthalmus & Alserio Lake & Galli et al., 2002 \\
\hline Dactylogyrus dulkeiti Bychowsky, 1936 & Carassius carassius & Varese Lake & None \\
\hline \multirow[t]{4}{*}{ Dactylogyrus ergensi Molnar, 1964} & Leuciscus cephalus & Lambro River & Galli et al., 2002 \\
\hline & Leuciscus cephalus & Ticino River & Galli et al., 2002 \\
\hline & Leuciscus cephalus & Po River & Molnar and Ghittino, 1977 \\
\hline & Telestes souffia muticellus & Ticino River & Galli et al., 2002 \\
\hline \multirow[t]{2}{*}{ Dactylogyrus extensus Mueller et Van Cleave, 1932} & Cyprinus carpio & Ticino River & Galli et al., 2002 \\
\hline & Cyprinus carpio & Po River & Molnar and Ghittino, 1977 \\
\hline Dactylogyrus formosus Kulwiec, 1927 & Carassius carassius & Varese Lake & Galli et al., 2003 \\
\hline Dactylogyrus rutili Gläser, 1965 & Rutilus aula & Como Lake & Galli et al., 2002 \\
\hline Dactylogyrus prostae Molnar, 1964 & Leuciscus cephalus & Po River & Molnar and Ghittino, 1977 \\
\hline Dactylogyrus sphyrna Linstow, 1878 & Rutilus pigus & Como Lake & None \\
\hline Dactylogyrus squameus Gussev, 1955 & Pseudorasbora parva & Ticino River & None \\
\hline Dactylogyrus tincae Gussev, 1965 & Tinca tinca & Ticino River & Galli et al., 2002 \\
\hline Dactylogyrus vastator Nybelin, 1924 & Carassius carassius & Varese Lake & Galli et al., 2002 \\
\hline \multirow[t]{3}{*}{ Dactylogyrus vistulae Prost, 1957} & Leuciscus cephalus & Lambro River & Galli et al., 2002 \\
\hline & Leuciscus cephalus & Ticino River & Galli et al., 2002 \\
\hline & Telestes souffia muticellus & Ticino River & None \\
\hline Dactylogyrus zandti Bychowsky, 1933 & Abramis brama & Ticino River & None \\
\hline Pseudodactylogyrus anguillae (Yin et Sproston, 1948) & Anguilla anguilla & Ticino River & Pretti et al. 2002 \\
\hline Pseudodactylogyrus bini (Kikuchi, 1929) & Anguilla anguilla & Ticino River & Pretti et al. 2002 \\
\hline Cleidodiscus pricei Muller, 1936 & Ictalurus melas & Varese Lake & Galli et al., 2002 \\
\hline \multirow[t]{2}{*}{ Actinocleidus oculatus (Mueller, 1934) } & Lepomis gibbosus & Alserio Lake & Galli et al., 2003 \\
\hline & Lepomis gibbosus & Ticino River & Galli et al., 2003 \\
\hline Actinocleidus recurvatus Mizelle et Donahue, 1944 & Lepomis gibbosus & Alserio Lake & Galli et al., 2003 \\
\hline Urocleidus dispar (Mueller, 1936) & Lepomis gibbosus & Ticino River & Galli et al., 2003 \\
\hline \multirow[t]{3}{*}{ Urocleidus similis (Mueller, 1936) } & Lepomis gibbosus & Alserio Lake & Galli et al., 2003 \\
\hline & Lepomis gibbosus & Como Lake & Galli et al., 2003 \\
\hline & Lepomis gibbosus & Ticino River & Galli et al., 2003 \\
\hline Onchocleidus principalis Mizelle, 1936 & Micropterus salmoides & Adda River & None \\
\hline Thaparocleidus vistulensis (Siwak, 1932) Lim, 1996 & Silurus glanis & Ticino River & Galli et al., 2003 \\
\hline \multicolumn{4}{|l|}{ Tetraonchidae } \\
\hline Tetraonchus monenteron (Wagener, 1857) & Esox lucius & Ticino River & Galli et al., 2003 \\
\hline \multicolumn{4}{|l|}{ Gyrodactylidae } \\
\hline Gyrodactylus anguillae Ergens, 1960 & Anguilla anguilla & Tiber River & Orecchia et al., 1987 \\
\hline Gyrodactylus carassii Malmberg, 1957 & Telestes souffia muticellus & Ticino River & None \\
\hline \multirow[t]{2}{*}{ Gyrodactylus gasterostei Gläser, 1974} & Rutilus aula & Como Lake & Galli et al., 2002 \\
\hline & Rutilus aula & Ticino River & None \\
\hline Gyrodactylus katharineri Malmberg, 1964 & Cyprinus carpio & Villoresi Cannal & Galli et al., 2002 \\
\hline Gyrodactylus lucii Kulakowskaja, 1951 & Esox lucius & Ticino River & Galli et al., 2002 \\
\hline Gyrodactylus sprostonae Lin Mo-en, 1962 & Cyprinus carpio & Po River & Molnar and Ghittino, 1977 \\
\hline Gyrodactylus tincae Malmberg, 1957 & Rutilus aula & Ticino River & None \\
\hline \multicolumn{4}{|l|}{ Diplozoidae } \\
\hline \multicolumn{4}{|l|}{ Paradiplozoon megan Molnar, 1964 (Quoted as } \\
\hline Paradiplozoon rutili in Galli et al., 2002). & Leuciscus cephalus & Lambro River & Galli et al., 2002 \\
\hline
\end{tabular}

2004. Fish were sampled using backpack electrofishing and carried fresh to the laboratory where their gills and fins were removed and examined under a stereomicroscope at magnifications ranging from $\times 4$ to $\times 20$. Monogenoids were mounted by flattening and fixed with ammonium picrate and glycerin (Malmberg, 1970). For Paradiplozoon, the same methods were used after cutting the opisthaptor. Parasites were identified using a differential interference contrast microscope with an attached camera lucida. Voucher specimens of new locality records were deposited in the para- 
Table 2. Cross comparison of parasitological data about both native and alien hosts. Hosts: N, native; A, alien; E, endemic. Monogenoids: a, alien (monogenoids detected only on alien hosts); $n$, native (monogenoids found only in native fish); u, unknown (monogenoids collected both in native and alien fish). References of monogenoid records are reported in brackets.

\begin{tabular}{|c|c|c|c|c|c|}
\hline \multicolumn{4}{|c|}{ Italian records } & \multicolumn{2}{|c|}{ Records from literature (Italy and foreign countries) } \\
\hline Host & Status & Parasites & Status & $\begin{array}{l}\text { Alien host } \\
\text { (referred to Italy) }\end{array}$ & $\begin{array}{l}\text { Native host } \\
\text { (referred to Italy) }\end{array}$ \\
\hline \multicolumn{6}{|l|}{ Cyprinidae } \\
\hline Abramis brama & A & Dactylogyrus zandti & A & $\begin{array}{l}\text { Blicca bjoerkna (Moravec, 2001) } \\
\text { Rutilus rutilus (Gussev, 1985) }\end{array}$ & \\
\hline \multirow[t]{4}{*}{ Carassius carassius } & A & Dactylogyrus anchoratus & $\mathrm{U}$ & $\begin{array}{l}\text { Barbus capito conocephalus } \\
\quad \text { (Gussev, 1985) } \\
\text { Carassius auratus (Dove } \\
\quad \text { and Ernst, 1998) } \\
\text { Cyprinus carpio (Gussev, 1985) } \\
\text { Labeo niloticus (Hassanein } \\
\text { et al., 1997) } \\
\text { Leucaspius delineatus } \\
\quad \text { (Moravec, 2001) }\end{array}$ & $\begin{array}{l}\text { Gobio gobio (Moravec, } \\
\text { 2001) }\end{array}$ \\
\hline & & Dactylogyrus dulkeiti & A & $\begin{array}{l}\text { Carassius auratus gibelio } \\
\text { (Gussev, 1985) } \\
\text { Cyprinus carpio (Kritsky } \\
\text { and Heckmann, 2002) }\end{array}$ & \\
\hline & & Dactylogyrus formosus & $\mathrm{a}$ & $\begin{array}{l}\text { Carassius auratus gibelio } \\
\text { (Gussev, 1985) } \\
\text { Cyprinus carpio (Kritsky } \\
\text { and Heckmann, 2002) }\end{array}$ & \\
\hline & & Dactylogyrus vastator & $\mathrm{a}$ & $\begin{array}{l}\text { Barbus barbus (Moravec, 2001) } \\
\text { Carassius auratus gibelio } \\
\quad \text { (Gussev, 1985) } \\
\text { Cyprinion watsoni (Jan } \\
\text { and Khan, 2001) } \\
\text { Cyprinus carpio (Gussev, 1985) }\end{array}$ & \\
\hline \multirow[t]{3}{*}{ Cyprinus carpio } & A & Dactylogyrus extensus & $\mathrm{a}$ & $\begin{array}{l}\text { Barbus graellsii } \\
\quad \text { (Gutierrez-Galindo and } \\
\text { Lacasa-Millan, 2001) } \\
\text { Carassius carassius (Simon } \\
\text { Vicente et al., 1975) } \\
\text { Cyprinus carpio haematopterus } \\
\text { (Gussev, 1985) } \\
\text { Misgurnus fossilis (Moravec, 2001) } \\
\text { Schilbe mystus (Hassanein } \\
\text { et al., 1997) }\end{array}$ & \\
\hline & & Gyrodactylus katharineri & $\mathrm{u}$ & $\begin{array}{l}\text { Barbus barbus (Gelnar, 1987) } \\
\text { Barbus graellsii } \\
\quad \text { (Gutierrez-Galindo and } \\
\text { Lacasa-Millan, 2001) } \\
\text { Barbus meridionalis } \\
\quad \text { petenyi (Prost, 1988) }\end{array}$ & $\begin{array}{l}\text { Gobio gobio (Ergens } \\
\text { and Lom, 1970) }\end{array}$ \\
\hline & & Gyrodactylus sprostonae & a & $\begin{array}{l}\text { Carassius auratus gibelio } \\
\quad \text { (Moravec, 2001) }\end{array}$ & \\
\hline \multirow[t]{4}{*}{ Leuciscus cephalus } & $\mathrm{N}$ & Paradiplozoon megan & $\mathrm{u}$ & Leuciscus idus (Gussev, 1985) & \\
\hline & & Dactylogyrus ergensi & $\mathrm{u}$ & $\begin{array}{l}\text { Chondrostoma nasus (Dupont } \\
\text { and Lambert, 1986) } \\
\text { Chondrostoma kneri } \\
\text { (Ergens, 1970) }\end{array}$ & $\begin{array}{l}\text { Leuciscus cephalus } \\
\quad \text { (Galli et al., 2002) } \\
\text { Telestes muticellus } \\
\quad \text { (Galli et al., 2002) }\end{array}$ \\
\hline & & Dactylogyrus prostae & $\mathrm{n}$ & & $\begin{array}{l}\text { Leuciscus cephalus } \\
\quad \text { (Stojanovski et al., 2004) }\end{array}$ \\
\hline & & Dactylogyrus vistulae & $\mathrm{u}$ & $\begin{array}{l}\text { Abramis bjoerkna (Moravec, 2001) } \\
\text { Alburnoides bipunctatus } \\
\quad \text { (Ergens, 1970) }\end{array}$ & $\begin{array}{l}\text { Alburnus alburnus (Dupont } \\
\text { and Lambert, 1986) }\end{array}$ \\
\hline
\end{tabular}


Table 2. Continued.

\begin{tabular}{|c|c|c|c|c|c|}
\hline \multicolumn{4}{|c|}{ Italian records } & \multicolumn{2}{|c|}{ Records from literature (Italy and foreign countries) } \\
\hline Host & Status & Parasites & Status & $\begin{array}{l}\text { Alien host } \\
\text { (referred to Italy) }\end{array}$ & $\begin{array}{l}\text { Native host } \\
\text { (referred to Italy) }\end{array}$ \\
\hline & & & & $\begin{array}{l}\text { Chalcalburnus chalcoides } \\
\text { (Molnar and Jalali, 1992) }\end{array}$ & $\begin{array}{l}\text { Scardinius erythrophthalmus } \\
\quad \text { (Moravec, 2001) }\end{array}$ \\
\hline & & & & $\begin{array}{l}\text { Chondrostoma nasus (Dupont } \\
\text { and Lambert, 1986) }\end{array}$ & \\
\hline & & & & $\begin{array}{l}\text { Chondrostoma nasus prespensis } \\
\text { (Stojanovski et al., 2004) }\end{array}$ & \\
\hline & & & & $\begin{array}{l}\text { Ctenopharyngodon idella } \\
\quad \text { (Moravec, 2001) }\end{array}$ & \\
\hline & & & & $\begin{array}{l}\text { Leuciscus idus (Ondrakova } \\
\text { et al., 2004) }\end{array}$ & \\
\hline & & & & Leuciscus leuciscus (Moravec, 2001) & \\
\hline & & & & $\begin{array}{l}\text { Pseudorasbora parva } \\
\quad \text { (Ondrakova et al., 2004) }\end{array}$ & \\
\hline & & & & Rutilus pigus (Moravec, 2001) & \\
\hline & & & & Rutilus rutilus (Moravec, 2001) & \\
\hline & & & & Vimba vimba (Moravec, 2001) & \\
\hline Pseudorasbora parva & A & Dactylogyrus squameus & a & - & \\
\hline \multirow[t]{12}{*}{ Rutilus aula } & $\mathrm{E}$ & Dactylogyrus & $\mathrm{u}$ & Leuciscus idus (Gussev, 1985) & \\
\hline & & alatus f. typica & & $\begin{array}{l}\text { Leuciscus idus oxianus } \\
\quad \text { (Gussev, 1985) }\end{array}$ & \\
\hline & & & & Leuciscus leuciscus (Gussev, 1985) & \\
\hline & & & & $\begin{array}{l}\text { Leuciscus leuciscus baicalensis } \\
\quad \text { (Gussev, 1985) }\end{array}$ & \\
\hline & & & & $\begin{array}{l}\text { Rutilus rutilus lacustris } \\
\quad \text { (Gussev, 1985) }\end{array}$ & \\
\hline & & Dactylogyrus caballeroi & $\mathrm{u}$ & - & \\
\hline & & Dactylogyrus rutili & $\mathrm{u}$ & Rutilus rutilus (Gussev, 1985) & \\
\hline & & Gyrodactylus gasterostei & $\mathrm{u}$ & $\begin{array}{l}\text { Gasterosteus aculeatus } \\
\quad \text { (Gussev, 1985) }\end{array}$ & $\begin{array}{l}\text { Leuciscus cephalus } \\
\quad \text { (Gussev, 1985) }\end{array}$ \\
\hline & & & & Blicca bjoerkna (Gussev, 1985) & Perca fluviatilis \\
\hline & & & & Pungitius pungitius (Gussev, 1985) & (Gussev, 1985) \\
\hline & & & & Rutilus rutilus (Gussev, 1985) & \\
\hline & & Gyrodactylus tincae & $\mathrm{u}$ & Tinca tinca $($ Gussev, 1985) & \\
\hline \multirow[t]{17}{*}{ Rutilus pigus } & A & Dactylogyrus crucifer & $\mathrm{u}$ & Abramis brama (Gussev, 1985) & Alburnus alburnus \\
\hline & & & & Blicca bjoerkna (Gussev, 1985) & (Gussev, 1985) \\
\hline & & & & Carassius auratus (Moravec, 2001) & Scardinius erythrophthalmus \\
\hline & & & & Leuciscus idus (Moravec, 2001) & (Gussev, 1985) \\
\hline & & & & Rutilus rutilus (Gussev, 1985) & $\begin{array}{l}\text { Leuciscus cephalus } \\
\text { (Moravec, 2001) }\end{array}$ \\
\hline & & Dactylogyrus sphyrna & $\mathrm{u}$ & Abramis brama (Gussev, 1985) & Alburnus alburnus (Dupont \\
\hline & & & & Barbus cyclolepis prespensis & and Lambert, 1986) \\
\hline & & & & (Dupont and Lambert, 1986) & Leuciscus cephalus \\
\hline & & & & Blicca bjoerkna (Gussev, 1985) & (Gussev, 1985) \\
\hline & & & & $\begin{array}{l}\text { Chondrostoma nasus } \\
\quad \text { (Moravec, 2001) }\end{array}$ & \\
\hline & & & & Leuciscus idus (Moravec, 2001) & \\
\hline & & & & $\begin{array}{l}\text { Leuciscus persidis (Jalali } \\
\quad \text { et al., 2000) }\end{array}$ & \\
\hline & & & & Rhodeus sericeus (Gussev, 1985) & \\
\hline & & & & Rutilus frisii kutum (Gussev, 1985) & \\
\hline & & & & Rutilus rutilus (Gussev, 1985) & \\
\hline & & & & Vimba vimba (Gussev, 1985) & \\
\hline & & & & $\begin{array}{l}\text { Rutilus Rubilio (Stojanovski } \\
\quad \text { et al., 2004) }\end{array}$ & \\
\hline \multirow[t]{2}{*}{$\begin{array}{l}\text { Scardinius } \\
\quad \text { erythrophthalmus }\end{array}$} & $\mathrm{N}$ & Dactylogyrus difformis & $\mathrm{u}$ & Blicca bjoerkna (Moravec, 2001) & $\begin{array}{r}\text { Leuciscus cephalus } \\
\text { (Moravec, 2001) }\end{array}$ \\
\hline & & & & Rutilus rutilus (Moravec, 2001) & \\
\hline
\end{tabular}


Table 2. Continued.

\begin{tabular}{|c|c|c|c|c|c|}
\hline \multicolumn{4}{|c|}{ Italian records } & \multicolumn{2}{|c|}{ Records from literature (Italy and foreign countries) } \\
\hline Host & Status & Parasites & Status & $\begin{array}{c}\text { Alien host } \\
\text { (referred to Italy) }\end{array}$ & $\begin{array}{l}\text { Native host } \\
\text { (referred to Italy) }\end{array}$ \\
\hline \multirow[t]{4}{*}{$\begin{array}{l}\text { Telestes souffia } \\
\text { muticellus }\end{array}$} & $\mathrm{N}$ & Dactylogyrus ergensi & $\mathrm{u}$ & $\begin{array}{l}\text { Chondrostoma colchicum } \\
\quad \text { kubanicum (Gussev, 1985) } \\
\text { Chondrostoma cyri (Gussev, 1985) } \\
\text { Chondrostoma oxyrhynchum } \\
\quad \text { (Gussev, 1985) } \\
\text { Chondrostoma kneri (Ergens, 1970) } \\
\text { Chondrostoma nasus (Gussev, 1985) }\end{array}$ & $\begin{array}{l}\text { Leuciscus cephalus (Galli } \\
\quad \text { et al., 2002) }\end{array}$ \\
\hline & & Dactylogyrus vistulae & $\mathrm{u}$ & $\begin{array}{l}\text { Alburnoides bipunctatus } \\
\quad \text { (Moravec, 2001) } \\
\text { Blicca bjoerkna (Moravec, 2001) } \\
\text { Chalcalburnus chalcoides } \\
\quad \text { (Molnar and Jalali, 1992) }\end{array}$ & $\begin{array}{l}\text { Alburnus alburnus } \\
\quad \text { (Moravec, 2001) } \\
\text { Leuciscus cephalus } \\
\quad \text { (Gussev, 1985) } \\
\text { Scardinius erythophthalmus } \\
\quad \text { (Moravec, 2001) }\end{array}$ \\
\hline & & & & $\begin{array}{l}\text { Chondrostoma nasus Gussev, 1985) } \\
\text { Ctenopharyngodon idella } \\
\quad \text { (Moravec, 2001) } \\
\text { Leuciscus idus (Ondrakova } \\
\quad \text { et al., 2004) } \\
\text { Leuciscus leuciscus (Moravec, 2001) } \\
\text { Pseudorasbora parva } \\
\quad \text { (Ondrakova et al., 2004) } \\
\text { Rutilis rutilus (Moravec, 2001) } \\
\text { Rutilus pigus (Moravec, 2001) } \\
\text { Vimba vimba (Moravec, 2001) }\end{array}$ & \\
\hline & & Gyrodactylus carassii & $\mathrm{u}$ & $\begin{array}{l}\text { Carassius auratus (Moravec, 2001) } \\
\text { Carassius carassius (Gussev, 1985) } \\
\text { Leucaspidus delineatus } \\
\quad \text { (Moravec, 2001) } \\
\text { Leuciscus leuciscus (Moravec, 2001) } \\
\text { Rutilus rutilus (Gussev, 1985) }\end{array}$ & $\begin{array}{l}\text { Alburnus alburnus } \\
\text { (Gussev, 1985) } \\
\text { Leuciscus cephalus } \\
\quad \text { (Gussev, 1985) } \\
\text { Scardinius } \\
\text { erythrophthalmus } \\
\text { (Gussev, 1985) }\end{array}$ \\
\hline Tinca tinca & A & Dactylogyrus tincae & a & & \\
\hline \multicolumn{6}{|l|}{ Anguillidae } \\
\hline \multirow[t]{3}{*}{ Anguilla anguilla } & $\mathrm{N}$ & Gyrodactylus anguillae & $\mathrm{n}$ & $\begin{array}{l}\text { Anguilla reinhardtii (Ernst } \\
\text { et al., 2000) } \\
\text { Anguilla australis (Ernst } \\
\quad \text { et al., 2000) } \\
\text { Anguilla japonica (Gussev, } \\
\text { 1985) } \\
\text { Anguilla rostrata (Crane } \\
\text { and Eversole, 1985) }\end{array}$ & \\
\hline & & $\begin{array}{l}\text { Pseudodactylogyrus } \\
\text { anguillae }\end{array}$ & $\mathrm{a}$ & $\begin{array}{l}\text { Anguilla japonica (Kennedy } \\
\text { and Di Cave, 1998) } \\
\text { Anguilla rostrata (Barker } \\
\quad \text { and Cone, 2000) }\end{array}$ & \\
\hline & & Pseudodactylogyrus bini & a & $\begin{array}{l}\text { Anguilla rostrata (Barker } \\
\text { and Cone, 2000) } \\
\text { Anguilla japonica (Kennedy } \\
\text { and Di Cave, 1998) }\end{array}$ & \\
\hline \multicolumn{6}{|l|}{ Ictaluridae } \\
\hline Ictalurus melas & A & Cleidodiscus pricei & $\mathrm{a}$ & $\begin{array}{l}\text { Ictalurus nebulosus } \\
\quad \text { (Kiskaroly, 1977) } \\
\text { Ictalurus punctatus } \\
\quad \text { (Chernova et al., 1988) } \\
\text { Ictalurus platycephalus } \\
\quad \text { (Cloutman, 1978) }\end{array}$ & \\
\hline
\end{tabular}


Table 2. Continued.

\begin{tabular}{|c|c|c|c|c|c|}
\hline \multicolumn{4}{|c|}{ Italian records } & \multicolumn{2}{|c|}{ Records from literature (Italy and foreign countries) } \\
\hline Host & Status & Parasites & Status & $\begin{array}{c}\text { Alien host } \\
\text { (referred to Italy) }\end{array}$ & $\begin{array}{l}\text { Native host } \\
\text { (referred to Italy) }\end{array}$ \\
\hline & & & & $\begin{array}{l}\text { Ictalurus catus (Hensley } \\
\text { and Nahhas, 1975) }\end{array}$ & \\
\hline \multicolumn{6}{|l|}{ Centrarchidae } \\
\hline \multirow[t]{4}{*}{ Lepomis gibbosus } & A & Actinocleidus oculatus & a & - & \\
\hline & & Actinocleidus recurvatus & a & - & \\
\hline & & Urocleidus dispar & a & $\begin{array}{l}\text { Lepomis macrochirus } \\
\text { (Rawson and Rogers, } \\
\text { 1972) }\end{array}$ & \\
\hline & & Urocleidus similis & $\mathrm{a}$ & - & \\
\hline Micropterus salmoides & A & Onchocleidus principalis & a & - & \\
\hline \multicolumn{6}{|l|}{ Siluridae } \\
\hline Silurus glanis & A & Thaparocleidus vistulensis & a & - & \\
\hline \multicolumn{6}{|l|}{ Esocidae } \\
\hline \multirow[t]{5}{*}{ Esox lucius } & $\mathrm{N}$ & Gyrodactylus lucii & $\mathrm{u}$ & $\begin{array}{l}\text { Stizostedion lucioperca } \\
\text { (Moravec, 2001) }\end{array}$ & $\begin{array}{l}\text { Perca fluviatilis } \\
\qquad \text { (Moravec, 2001) }\end{array}$ \\
\hline & & Tetraonchus monenteron & $\mathrm{u}$ & $\begin{array}{l}\text { Chondrostoma nasus } \\
\text { (Moravec, 2001) }\end{array}$ & $\begin{array}{r}\text { Leuciscus cephalus } \\
\text { (Moravec, 2001) }\end{array}$ \\
\hline & & & & Cyprinus carpio (Moravec, 2001) & Scardinius \\
\hline & & & & $\begin{array}{l}\text { Leuciscus leuciscus } \\
\quad \text { (Moravec, 2001) }\end{array}$ & $\begin{array}{l}\text { erythrophthalmus } \\
\text { (Moravec, 2001) }\end{array}$ \\
\hline & & & & Rutilus rutilus (Moravec, 2001) & $\begin{array}{l}\text { Gobio gobio } \\
\quad \text { (Moravec, 2001) }\end{array}$ \\
\hline
\end{tabular}

sitological collection of the Museo di Storia Naturale di Milano, Italy (MSNM Pi: 4417-8: Gyrodactylus tincae; MSNM Pi 4419-35: Gyrodactylus carassii; MSNM Pi 4436-51: Gyrodactylus gastestorei; MSNM Pi 4452: Dactylogyrus alatus; MSNM Pi 4453-503: Dactylogyrus caballeroi; MSNM Pi 4504-38: Dactylogyrus vistulae; MSNM Pi 4539-40: Dactylogyrus squameus; MSNM Pi 4541-65: Dacylogyrus sphyrna; MSNM Pi 4566-93: Onchocleidus principalis; MSNM Pi 4594-96: Dactylogyrus crucifer; MSNM Pi 4597: Dactylogyrus dulkeiti; MSNM Pi 4598-601: Dactylogyrus zandti).

Host species were ranked into three categories according to fishbase (Froese and Pauly; 2005): native (N), originally from Italy but also present in other countries; alien (A), not originally from Italy, and endemic (E), originally from and found exclusively in Italy.

As the knowledge concerning original distribution of monogenoids is not complete, we propose cross-comparison of parasitological data about both native and alien hosts. We consider alien (a) the monogenoids detected only on alien hosts, and native (n) the monogenoids found only on native fish. Monogenoids collected from both native and alien fish are regarded as of unknown origin (u). This could be a reasonable instrument to verify if translocation of alien hosts includes their monogenoidean parasites, and if these parasites are able to colonize native hosts. Host specificity of monogenoids was checked using a keyword search on the CAB Abstracts and Medline (years 1966-2005) and consulting Gussev (1985), Bona et al. (1995), and Moravec (2001).

\section{RESULTS}

A total of 35 monogenoidean species was found on 16 species of Italian freshwater fish and a complete checklist of parasites with their hosts, location, and records data was compiled (Table 1). Among the 35 parasite species, 15 are new locality records in Italy.

Locality records of monogenoids from native hosts are compared with monogenoids from alien hosts in Table 2. According to the literature data, 11 fish species among the 16 listed can be considered alien, 4 native, and 1 endemic. Comparison between these data and the checklist suggests that 17 of the 35 monogenoidean species present in Italy should be considered as alien, 2 as native, and the remaining 16 of unknown origin.

\section{DISCUSSION}

Italian freshwater fish communities are undergoing rapid evolution as a consequence of the introduction of alien species. Besides considering an alien fish as a potential threat to native fish fauna in terms of competition (Bianco and Ketmaier, 2001), these aliens should be considered as a potential vehicle for the introduction of their symbiota organisms.

Alien monogenoids introduced to Italian fresh- 
waters with their alien host species are widespread: in fact, $49 \%$ of the monogenoids should be considered alien, whereas just 2 species are to be reasonably considered native. Lack of good historical knowledge regarding the original distribution of monogenoids leads us to consider the remaining species of unknown origin. For parasites of the aquacultured species Anguilla anguilla, we considered Gyrodactylus anguillae as native, while we considered Pseudodactylogyrus anguillae and Pseudodactylogyrus bini as alien, in agreement with Kennedy and Di Cave (1998).

The study of parasitofauna can be used for the investigation of the origin of host species. For example, there are divergent opinions about the origin of Carassius carassius, which is considered alien by some authors (Alessio and Gandolfi, 1983) and native by others (Tortonese, 1970; Blanc et al., 1971). Since we found Dactylogyrus dulkeiti, Dactylogyrus formosus, and Dactylogyrus vastator on it, and these are known from literature to be parasites only on exotic fish, we considered them as alien. Their presence supports the hypothesis of Alessio and Gandolfi (1983).

In some cases we are able to infer original geographic distribution of monogenoids from their host translocations: 5 species of monogenoids arrived in Italy from America (Actinocleidus oculatus, Actinocleidus recurvatus, Urocleidus dispar, Urocleiuds similis, and Onchocleidus principalis, 11 from Central Europe (Dactylogyrus zandti, D. dulkeiti, D. formosus, D. vastator, Dactylogyrus extensus, Gyrodactylus sprostonae, Dactylogyrus squameus, Dactylogyrus tincae, P. anguillae, P. bini, and Thaparocleidus vistulensis), and 1 from Eurasia (Dactylogyrus squameus).

The checklist provided in this paper should be considered as the most complete report of monogenoids of Italian freshwater fish. An average of 2.3 monogenoids was collected on each host species. Considering that the total number of Italian freshwater fish species is 82 , the total number of monogenoids in Italian freshwaters is estimated to be approximately 189 . This number will probably increase proportionally as more alien fish species are introduced to Italian freshwaters.

\section{LITERATURE CITED}

Alessio, G., and G. Gandolfi. 1983. Censimento e distribuzione attuale delle specie ittiche nel bacino del fiume Po. Quaderni dell'Istituto di Ricerca sulle Acque 67. C.N.R., Roma. 92 pp.

Amori, G., F. M. Angelici, S. Frugis, G. Gandolfi, R. Groppali, B. Lanza, G. Relini, and G. Vicini. 1993. Vertebrata. In A. Minelli, S. Ruffo, and S. La
Posta, eds. Checklist delle specie della fauna italiana Edizioni Calderini, Bologna, Italy. Fascicoli 1-110.

Arndt, G. M., J. Gessner, and C. Raymakers. 2002. Trends in farming, trade and occurrence of native and exotic sturgeons in natural habitats in Central and Western Europe. Journal of Applied Ichthyology 18: 444-448.

Barker, D. E., and D. K. Cone. 2000. Occurrence of Ergasilus celestis (Copepoda) and Pseudodactylogyrus anguillae (Monogenea) among wild eels (Anguilla rostrata) in relation to stream flow, $\mathrm{pH}$ and temperature and recommendations for controlling their transmission among captive eels. Aquaculture 187:261-274.

Bauer, O. N. 1991. Spread of parasites and diseases of aquatic organism by acclimatization: a short review. Journal of Fish Biology 39:679-686.

Bauer, O. N., N. O. Pugachev, and V. N. Voronin. 2002. Study of parasites and diseases of sturgeons in Russia: a review. Journal of Applied Ichthyology. 18:420-429.

Bianco, P. G. 1998. Freshwater fish transfer in Italy: history, local changes in fauna and a prediction on the future of native populations. In I. G. Cowx, ed. 1998. Stocking and introduction of fish. Oxford: Fishing News Books, Blackwell Science, UK. 456 pp.

Bianco, P. G., and V. Ketmaier. 2001. Anthropogenic changes in the freshwater fish fauna of Italy, with reference to the central region and Barbus graellsii, a newly established alien species of Iberian origin. Journal of Fish Biology 59:190-208.

Blanc, M., J. L. Gaudet, P. Banarescu, and J. C. Hureau. 1971. European Inland Water Fish. A Multilingual Catalogue. FAO, Fishing News Ltd, London, England. 196 pp.

Bona, F., E. Buriola, S. Cerioni, P. Orecchia, and L. Paggi. 1995. Digenea. In A. Minelli, S. Ruffo, and S. La Posta, eds. Checklist delle specie della fauna italiana, Fascicolo 5. Edizioni Calderini, Bologna, Italy. $31 \mathrm{pp}$.

Chernova, T. N., I. G. Mamyshev, and N. O. Spiranti. 1988. Parasite fauna of Ictalurus punctatus cultured on fish farms in the Georgian SSR. Bolezni i parazity v teplovodnom rybnom khozyaistve. Donish, Dushanbe, USSR. 86-88.

Cloutman, D. C. 1978. Abundance of Cleidodiscus pricei Mueller (Monogenea: Dactylogyridae) on the flat bullhead, Ictalurus platycephalus (Girard), in Lake Norman, North Carolina. Journal of Parasitology 64(1): 170-172.

Crane, J. S., and A. G. Eversole. 1985. Metazoan ectoparasitic fauna of American eels from brackish water. Pages 248-254 in Proceedings of the 39th Annual Conference, Southeastern Association of Fish and Wildlife Agencies, October 27-30, 1985, Lexington, Kentucky.

Dogiel, V. A. 1964. General Parasitology. Oliver and Boid, Edinburgh, U.K. 516 pp.

Dove, A. D. M., and I. Ernst. 1998. Concurrent invadersfour exotic species of Monogenea now established on exotic freshwater fishes in Australia. International Journal for Parasitology 28(11):1755-1764.

Dupont, F., and A. Lambert. 1986. Study of Dactylogyridae communities parasitic on Cyprinidae in Lake Mikri Presna (northern Greece). Description of three new species from an endemic Barbus $-B$. cyclolepis 
prespensis. Annales de Parasitologie Humaine et Comparée 61(6):597-616.

Eaton, J. G., and R. M. Scheller. 1996. Effects of climate warming on fish thermal habitat in streams of the United States. Limnology and Oceanography 41:1109_ 115 .

Ergens, R. 1970. Parasitic fauna of fish from Montenegro. I. Polyonchoinea (Monogenoidea) of some fish of Lakes Skadar and Veliko Crno. Pol'oprivreda i Shumarstvo 16:1-44.

Ernst, I., Fletcher, A., and C. Hayward. 2000. Gyrodactylus anguillae (Monogenea: Gyrodactylidae) from anguillid eels (Anguilla australis and Anguilla reinhardtii) in Australia: a native or an exotic? Journal of Parasitology 86:1152-1156.

Esch, G. W., and J. C. Fernández. 1993. A Functional Biology of Parasitism, Ecological and Evolutionary Implications. Chapman and Hall, London. 337 pp.

Froese, R., and D. Pauly, eds. 2005. FishBase. World Wide Web electronic publication. www.fishbase.org, accessed 10 November 2005.

Galli, P., G. Crosa, L. Mariniello, M. Ortis, and S. D'Amelio. 2001 Water quality as a determinant of fish parasite communities. Hydrobiologia 452: 173-179.

Galli, P., F. Stefani, F. Benzoni, G. Crosa, and A. Zullini. 2003. A. New records for Italy of alien monogeneans from Lepomis gibbosus and Silurus Glanis. Parassitologia 45(3-4):147-149.

Galli, P., F. Stefani, F, Benzoni, and A. Zullini. 2005. Introduction of alien host-parasite complexes in a natural environment and the symbiota concept. Hydrobiologia 548:293-299.

Galli, P., F. Stefani, S. Zaccara, and G. Crosa. 2002. Occurrence of Monogenea in Italian freshwater fish (Po river basin). Parassitologia 44:189-197.

Gattaponi, P., and C. Corallini Sorcetti. 1982. The parasitism and diet habitats of Scardinius erythrophthalmus in Lake Trasimeno. Convegno Società Italiana delle Scienze Veterinarie, Sanremo, Italy; 22-25 September.

Gelnar, M. 1987. Experimental verification of the effect of water temperature on micropopulation growth of Gyrodactylus katharineri Malmberg, 1964 (Monogenea) parasitizing carp fry (Cyprinus carpio L.). Folia Parasitologica 34:19-23.

Gussev, A. V. 1985. Order Dactylogyridae. Pages 15-251 in O. N. Bauer, ed. Key to the Parasites of Freshwater Fish Fauna of the USSR. Vol.2. (In Russian.)

Gutierrez-Galindo, J. F., and M. I. Lacasa-Millan. 2001. Study of the Monogenea of Cyprinidae in the Llobregat River, Northeastern Spain. II. Species composition on Barbus graellsii Steindachner, 1866. Research and Reviews in Parasitology 61:91-96.

Hassanein, M. A., S. I. Shalaby, A. Ashour, and N. ElAroussy. 1997. First record of some Monogenea from Nile fishes; with emphasis on some new hosts for these parasites. Bulletin of the National Research Centre (Cairo) 22(2):129-138.

Hensley, G. H., and F. M. Nahhas. 1975. Parasites of fishes from the Sacramento-San Joaquin Delta, California. California Fish and Game 61(4):201-208.

Jalali, B., M. Barzegar, and S. Shamsi. 2000. Occurrence and description of Dactylogyrus sphyrna Linstow,
1878 (Monogenea: Dactylogyride) on the gills of an Iranian endemic fish Leuciscus persidis Coad, 1981 as a new host. Iranian Journal of Fisheries Sciences 2:71-76.

Jan, A. H., and R. U. Khan. 2001. Prevalence of parasites in a fresh water cyprinid fish, Cyprinion watsoni from Ramli stream, Islamabad, Pakistan. Proceedings of Pakistan Congress of Zoology 21:323-330.

Kennedy, C. R., and D. Di Cave. 1998. Gyrodactylus anguillae (Monogenea): the story of an appearance and a disappearance. Folia Parasitologica 45:77-78.

Kiskaroly, M. 1977. Study of the parasite fauna of freshwater fishes from fish ponds of Bosnia and Herzegovina. A. Monogenean trematodes 1. I. Cyprinid fish ponds. Veterinaria (Sarajevo) 26(2/3):195-208.

Kritsky, D. C., and R. Heckmann. 2002. Species of Dactylogyrus (Monogenoidea: Dactylogyridae) and Trichodina mutabilis (Ciliata) infesting koi carp, Cyprinus carpio, during mass mMortality at a commercial rearing facility in Utah, U.S.A. Comparative Parasitology 69(2):217-218.

Lim, L. H. S. 1996. Thaparocleidus Jain, 1952, the senior synonym of Silurodiscoides Gussev, 1976 (Monogenea: Ancylodiscoidinae). Systematic Parasitology 35:207-215.

Malmberg, G. 1970. The excretory systems and the marginal hooks as basis for systematics of Gyrodactylus. Arkiv für Zoologie 23:1-235.

Molnar, K., and P. Ghittino. 1977. Alcuni vermi monogenoidea in pesci provenienti dal fiume Po e da piscicolture italiane. Rivista Italiana di Piscicoltura ed Ittiopatologia 12:110-111.

Molnar, K., and B. Jalali. 1992 Further monogeneans from Iranian freshwater fishes. Acta Veterinaria Hungarica 40:55-61

Moravec, F. 2001. Checklist of the metazoan parasites of fishes of the Czech Republic and the Slovak Republic (1873-2000). Academia Praha. 168 pp.

Ondrakova, M., I. Matejusova, A. Simkova, and M. Gelnar. 2004. New reports of dactylogyridean species (Monogenea) for Central Europe. Helminthologia 41(3):139-145.

Orecchia, P., M. Bianchini, N. Catalini, S. Cataudella, and L. Paggi. 1987. Parasitological study of a population of Tiber River eels (Anguilla anguilla). Parassitologia 29(1):37-47.

Pretti, C., G. Soldani, A. M. Cognetti-Varriale, G. Monni, V. Meucci, and L. Intorre. 2002. Efficacy and safety of azamethiphos for the treatment of pseudodactylogyrosis in the European eel. Journal of Veterinary Pharmacology and Therapeutics 25: 155-157.

Prost, M. 1988. Fish Monogenea of Poland. VIII. Parasites of Barbus meridionalis petenyi (Heck.). Acta Parasitologica Polonica 33:1-6.

Rawson, M. V., and W. A. Rogers. 1972. Seasonal abundance of ancyrocephalinaen (Monogenoidea) parasites of bluegill, Lepomis macrochirus (Raf.). Journal of Wildlife Diseases 8:255-260.

Simón Vicente, F., V. Ramajo Martín, and A. Encinas Grandes. 1975. Dactylogyrus spp. (Trematoda, Monogenea) de peces de la cuenca del río Duero. Revista Iberica de Parasitologia 35:25-40.

Stojanovski, S., Z. Kulisic, R. A. Baker, N. Hristovski, 
P. Cakic, and M. Hristovski. 2004. Fauna of monogenean trematodes-parasites of some cyprinid fishes from Lake Prespa (Macedonia). Acta Veterinaria 54: 73-82.

Torchin, M. E., K. D. Lafferty, A. P. Dobson, V. J. McKenzie, and A. M. Kuris. 2003. Introduced species and their missing parasites. Nature 421: 628-629.

Torchin, M. E., K. D. Lafferty, and A. M. Kuris. 2001. Release from parasites as natural enemies: increased performance of globally introduced marine crab. Biological Invasion 3:333-345.

Torchin, M. E., K. D. Lafferty, and A. M. Kuris. 2002.
Parasites and marine invasions. Parasitology 124: S137-S151.

Tortonese, E. 1970. Fauna d'Italia. XI, Osteichithyes, Pesci Ossei. Calderini Editore, Bologna, Italy. 545 pp.

Vitousek, P. M., C. M. D'Antonio, L. D. Loope, M. Rejmánek, and R. Westbrooks. 1997. Introduced species: a significant component of human-caused global change. New Zealand Journal of Ecology. 21: $1-16$.

Zerunian, S., and T. De Ruosi. 2002. Iconografia dei pesci delle acque interne d'Italia. Ministero dell'Ambiente e della Tutela del Territorio, Unione Zoologica Italiana. DCN, Roma. 259 pp. 